\title{
Will Statin Use Prevent Fracture of Type 2 Diabetes Associated with Thiazolidinedione Treatment? A Mini-Review and a Hypothesis
}

\author{
Chiung-Huei Peng ${ }^{\mathrm{a}}$, Yi-Sun Yang ${ }^{\mathrm{b}}$, Kuan-Chou Chen ${ }^{\mathrm{c}}$, Chiung-Chi Peng ${ }^{\mathrm{d}}$ and Chien-Ning Huang ${ }^{*}, \mathrm{~b}$ \\ ${ }^{a}$ Department of Nursing, Hungkuang University, Sha Lu, Taichung Hsien, Taiwan \\ ${ }^{b}$ Division of Endocrinology and Metabolism, Department of Internal Medicine, Chung-Shan Medical University \\ Hospital, Taichung City, Taiwan \\ ${ }^{c}$ Department of Urology, Taipei Medical University, Wu-Xin Street, Taipei City, Taiwan \\ ${ }^{d}$ Department of Nursing, Cardinal Tien's College of Nursing, Taipei Hsien, Taiwan
}

\begin{abstract}
Thiazolidinediones (TZDs) are a multi-effective anti-glycemic drug for type 2 diabetes. Recent clinical trials suggest TZDs are associated with bone loss or fracture in older diabetic women. Diabetic women exhibit more rapid bone loss despite the higher baseline bone mineral density. The higher fracture risk of type 2 diabetes may be associated with neural and vascular complications, or the fragile bone structure.

Statins were found to exert protective effects on bone through their anti-oxidative effect on vascular and advanced glycation end product-related disorders. The impact of combination use of statins and TZDs on bone however has never been addressed. Whereas considering the advantages of statins and TZDs in treating type 2 diabetes regarding their cardiovascular protection and glycemic control improvement, we hypothesize that the concomitant use of statins and TZDs might also prevent against bone complications of type 2 diabetes or induced by TZDs, especially for older diabetic women.
\end{abstract}

Keywords: Type 2 diabetes, bone fracture, thiazolidinediones, statins.

\section{INTRODUCTION}

Thiazolidinediones (TZDs) are one kind of effective oral anti-glycemic drugs (OAD) for type 2 diabetes. They promote insulin sensitivity via activating peroxisome proliferator activated receptor $\gamma$ (PPAR $\gamma$ ) and the subsequent regulation on adipocyte differentiation and fatty acid metabolism [1]. However, in A Diabetes Outcomes Progression Trial (ADOPT), rosiglitazone, one of the TZDs, is reported to be associated with bone loss and fracture in type 2 diabetic patients [2]. The chronic complications of diabetes are well known including the vasculopathy and neuropathy, whether osteo-dysregulation is induced by TZDs or by diabetes per se still remains unclear.

Among all the complications, coronary heart disease is the major cause of mortality in diabetic individuals. It is reported that the statin drug simvastatin effectively lowers the concentrations of low density lipoprotein C (LDL-C) and apoprotein B (apoB) in TZDs-treated type 2 diabetic patients [3]. Combinations of atorvastatin and rosiglitazone further decrease high sensitivity $\mathrm{C}$ reactive protein (hsCRP) level and increase adiponectin, and exert their effect on other biomarkers compared with the group treated with rosiglitazone alone [4]. Statins are recently suggested to have boneprotective effect [5]. However, the impact of combination use of statins and TZDs on bone has never been addressed.

In the present report, we review the evidence in association with diabetes, TZDs, statin and fracture, and hypothe-

\footnotetext{
*Address correspondence to this author at the Division of Endocrinology and Metabolism, Department of Internal Medicine, Chung-Shan Medical University Hospital, No. 110, Section 1, Chien-Kuo N. Road, Taichung City, Taiwan; Tel: 886-4-24739595, Ext. 34708; Fax: 886-4-24739220; E-mail: cshy049@csh.org.tw
}

size that a combination use of statins and TZDs might be beneficial to prevent bone loss or fracture in TZDs-treated type 2 diabetic patients.

\section{DIABETES ASSOCIATED WITH OSTEOPOROSIS OR BONE FRACTURE}

In the previous reports, both cross-sectional and prospective studies showed that type 1 diabetic patients were associated with the decrease in bone mineral density (BMD) and the increased risk of osteoporosis and bone fracture [6]. However, there exist controversies in the investigations of type 2 diabetes. Most studies demonstrated that type 2 diabetic patients had higher BMD, probably due to increased body weight, but others reported non-significant differences [7-9]. The cross-sectional studies reported decreased risk of fracture in type 2 diabetic patients, whereas prospective studies demonstrated that type 2 diabetes was associated with increased osteoporotic fracture, despite the higher BMD $[10,11]$. When compared diabetic individuals with or without fractures, the former were found to have lower hip BMD and lean mass, and were more likely to have peripheral neuropathy, ischemic stroke and falls, implying that other factors such as diabetic complications could be involved in the fracture risk [12].

Some reports demonstrated that there existed sex difference with osteoporosis in type 2 diabetic patient. Older women with type 2 diabetes had better BMD, whereas no differences were observed in men [13]. Schwartz et al. indicated that diabetic white women, but not men or black women, had the more rapid bone loss despite having the higher baseline BMD [14]. In Women's Health Initiative Observational Study (WHI-OS), women with type 2 diabetes showed to be at increased risk for fractures even after adjust- 
ing the visual problems and frequency of falls [15]. Although the bone density seems to be greater in diabetes, the bone structure could be more fragile caused by the formation of advanced glycation end (AGE) products, the lower levels of IGF-I, and hypercalciuria or inflammation [15].

\section{TZDS THERAPY AND BONE COMPLICATION}

TZDs have been permitted in clinical use since 1997. Since the hepatotoxicity induced by troglitazone, currently, only rosiglitazone and pioglitazone are used to treat type 2 diabetic patients [1]. TZDs bind to and activate PPAR $\gamma$, a member of steroid nuclear receptor family observed in adipose and other insulin target tissues, thus mediating adipogenesis and the expression of relating markers [16]. PPAR $\gamma$ is also expressed in bone marrow, where the mesenchymal cells could differentiate into adipocytes and osteoblasts, indicating the potential effect of TZDs in regulating bone metabolism $[17,18]$. Actually, TZD was reported to inhibit osteoclast-like cell formation and bone resorption in vitro, which was not related to its adipogenic effect [19]. TZD was reported to decrease bone turnover markers in 33 Japanese type 2 diabetic patients before significant improvement of glucose metabolism, suggesting the direct effect of TZD on bone [20]. In addition, TZD was shown to decrease serum leptin, which could be associated with preventing bone loss of type 2 diabetes [21].

However, recently, many studies have demonstrated the negative impact of TZDs on bone. In vitro studies demonstrate that PPAR $\gamma 2$ activation in marrow stroma cells increases adipocyte differentiation while decreasing osteoblastogenesis [22]. PPAR $\gamma 2$ enhances the expression of aP2, a marker of differentiated adipocytes, but reduces Runx2, the main regulator of osteoblasts. In addition, the activation of PPAR $\gamma$ also suppresses other markers involved in osteoblast differentiation, such as $\alpha 1$ ( I )collagen alkaline phosphatase, osteocalcin and osteopontin [23]. These evidences suggest that PPAR $\gamma$ may act as a switch point between osteogenic and adipogenic pathways. It was reported TZD increased the amount of bone marrow adipose tissue [24]. In rosiglitazonetreated mice, TZD was demonstrated to promote osteogenic cell apoptosis and decrease the trabecular volume and BMD [25]. On the contrary, PPAR $\gamma$ deficient mice exhibited enhanced osteogenesis [26]. In addition to the osteogenesis pathway, by using a Tie2Cre/flox mouse model, PPAR $\gamma$ deletion is demonstrated to impair osteoclastogenesis, thus resulting in osteopetrosis and extramedullary hematopoiesis, whereas rosiglitazone promotes osteoclast differentiation in a receptor-dependent manner [27].

Clinically, some prospective observational studies suggest that TZDs cause bone loss in older diabetic women [28]. Results of recent ADOPT showed that rosiglitazone use increased fracture risk of diabetic patients, whereas the fracture usually occured at humerus, hand and foot [2]. In a randomized controlled trial, rosiglitazone exerted skeletal effects by decreasing bone formation and BMD in healthy postmenopausal women, especially at hip and lumber [29]. Another popularly used TZD pioglitazone, was also advised to cause fracture risk of diabetic female in 2007 [30]. As diabetic women may increase bone turnover per se, it is of great possibility that TZDs superimposed the bone loss. In addition, TZDs are known to inhibit aromatase pathway, the main estrogen source of postmenopausal women, implicating another possible mechanism for TZDs to impact negatively on bone [28].

\section{STATINS AND THE POSSIBLE EFFECT ON BONE}

Statins are hydroxymethylglutaryl-coenzyme A (HMG$\mathrm{CoA}$ ) reductase inhibitors, which are wildly used to treat hypercholesteremia. Statins were demonstrated to prevent primary and secondary coronary artery disease in diabetic patients [31,32]. In recent years, statins were found to increase new bone formation in rodents and in human cells [5].

In vitro experiment of different cell lines showed that statins exerted their effects on stimulating osteoblast differentiation and bone formation via BMP-2 expression, and/ or inhibiting osteoclast differentiation and bone resorption [3336]. In vivo animal studies also suggested that statins were effective to increase bone formation or decrease bone resorption [37,38]. However, discrepancies exist in humans. In Geelong cross-sectional study, statin use was associated with $60 \%$ reduction of fracture risk, which was greater than would be expected from the increase of BMD [39]. Statins seemed to reduce the risk of fracture among older women [40]. In a prospective cohort study of Netherland, statins use was associated with lower risk of vertebral fracture. The relative risk decreased on higher cumulative use [41]. A Danish casecontrol study also suggested that statin use decreased the hip fracture [42]. In the previous investigations, it was demonstrated statins, but not the other lipid-lowering drugs, reduced the fracture risk $[43,44]$. The anabolic and antiresorptive effects of statins increased more BMD in postmenopausal women than those treated by hormone replacement alone [45]. However, results of recent WHI-OS suggested that statin use did not improve bone density and fracture risk [46]. Some systemic analysis reported that statins might be effective in improving hip BMD and lowering the risk of non-spine fractures [47]. Whereas in a randomized controlled trial, simvastatin showed no benefit on BMD for postmenopausal osteopenic women [48].

Although there still exist controversies about statinmediated bone effect, some reports indicate that statin increase BMD and prevent bone loss in patients with type 2 diabetes [49,50]. In the clinical trials, lovastatin is shown to prevent bone loss in postmenopausal diabetic women, whereas atorvastatin has no effect on bone turnover in type 2 diabetic patients [51,52].

The possible mechanism of statins has been investigated in in vitro studies. Mevalonate abolished statin-induced mineralization, suggesting the direct regulating role of HMGCoA on bone metabolism [53]. It was suggested that statins could integrate PI3K signal to Akt and MAPK thus mediate osteoblast differentiation via BMP-2 expression [54]. Furthermore, statins were reported to decrease the serum level of AGE $[55,56]$. In diabetic patients, the cross-linking and accumulation of AGE are associated with tissue damage such as vascular and neural complications. It was demonstrated that atorvastatin inhibited AGE-induced reaction by suppressing reactive oxygen species generation, which acted in a cholesterol-lowering independent manner, and could contribute to the early clinical benefit among diabetic patients [56]. In fact, the protective effect of statins on bone 
could include their impact on vascular disturbance and AGErelated disorders.

\section{HYPOTHESIS: COMBINATION USE OF STATINS WITH TZDs}

Fracture and cardiovascular disease are the common morbidity or mortality for ageing people. According to the previous reports mentioned above, statins might be expected to exert protective effects against the bone complication associated with TZDs or type 2 diabetes. TZDs used in type 2 diabetic treatment exert their clinical effects not only on improving insulin sensitivity, $\beta$-cell preservation, but also on the elevation of adiponectin and anti-inflammation [57,58]. Although it was once considered to be associated with increased myocardial infarction recently [59], another recent meta-analysis found no significantly increased risk of cardiovascular mortality by using either rosiglitazone or pioglitazone [60]. Furthermore, the longterm clinical trial, PROactive study, as well as a meta-analysis regarding pioglitazone on cardiovascular disease risk has also suggested that the drug demonstrated a protective effect [61,62]. ADOPT study, apparently, showed that TZDs act more effective to control hyperglycemia, and consistently maintain $\mathrm{HbA1C}$ level below 7.0\%. However, ADOPT also showed that TZDs increase the bone fracture risk of women [2].

It has been documented in the large scaled clinical trial such as HPS and CARDS that statin could reduce cardiovascular events for type 2 diabetic patients [31,32]. In addition, many previous reports suggested stain also prevent the risk of bone fracture. Hence we here hypothesize that combination therapy of TZD and statin for type 2 diabetic patients could improve glycemic control and protect cardiovascular system, as well as prevent potential bone loss or fracture induced by TZD or by diabetes per se. The important antiinflammation effect could also be provided by both medication. While concerning poly-pharmacy as a treatment limitation for patients, premixed with TZD and statin as a single tablet might be an option to improve drug compliance.

In conclusion, combination use of TZDs and statins could be expected to be the best treatment choice for type 2 diabetic patients, especially among postmenopausal women. Further studies in vitro, in vivo or clinical trials are still necessary to see the bone effect by the concomitant use of TZDs and statins.

\section{CONCLUSION}

- $\quad$ Diabetes per se could result in bone fracture via rapid bone loss, fragile bone structure, or due to vascular complications and neuropathy.

- $\quad$ TZDs are effective OAD which exert their effects not only on increasing insulin sensitivity, but on the pleiotropic functions including changing lipid profile and anti-inflammation. However, recent ADOPT study demonstrated TZDs increase the bone fracture risk of diabetic women.

- Many studies have shown that statins might be beneficial in preventing bone loss or decrease fracture risk. In literature they were shown to increase the BMD of type 2 diabetic patients.
Statins have been shown to reduce cardiovascular events for type 2 diabetic patients. Combination use of statins and TZDs effectively lower the concentrations of LDL-C, apoB and CRP, as well as increase adiponectin. Hence combination therapy with both statins and TZDs could be expected not only to improve glycemic control and protect cardiovascular system, but also to prevent potential bone loss or fracture induced by TZDs or by diabetes per se.

\section{REFERENCES}

[1] Yki-Jarvinen H. Thiazolidinediones. N Engl J Med 2004; 351: 1106-18.

[2] Kahn SE, Haffner SM, Heise MA, et al. Glycemic durability of rosiglitazone, metformin, or glyburide monotherapy. N Engl J Med 2006; 355: 2427-43.

[3] Lewin AJ, Kipnes MS, Meneghini LF, et al. Effects of simvastatin on the lipid profile and attainment of low-density lipoprotein cholesterol goals when added to thiazolidinedione therapy in patients with type 2 diabetes mellitus: A multicenter, randomized, doubleblind, placebo-controlled trial. Clin Ther 2004; 26: 379-89.

[4] Chu CS, Lee KT, Lee MY, et al. Effects of rosiglitazone alone and in combination with atorvastatin on nontraditional markers of cardiovascular disease in patients with type 2 diabetes mellitus. Am J Cardiol 2006; 97: 646-50.

[5] Mundy G, Garrett R, Harris S, et al. Stimulation of bone formation in vitro and in rodents by statins. Science 1999; 286: 1946-9.

[6] Lipscombe LL, Jamal SA, Booth GL, et al. The risk of hip fractures in older individuals with diabetes: a population-based study. Diabetes Care 2007; 30: 835-41.

[7] van Daele PL, Stolk RP, Burger H, et al. Bone density in noninsulin-dependent diabetes mellitus. The Rotterdam Study. Ann Intern Med 1995; 122: 409-14.

[8] Weinstock RS, Goland RS, Shane E, et al. Bone mineral density in women with type II diabetes mellitus. J Bone Miner Res 1989; 4: 97-101.

[9] Tuominen JT, Impivaara O, Puukka P, et al. Bone mineral density in patients with type 1 and type 2 diabetes. Diabetes Care 1999; 22: 1196-200.

[10] Heath H, 3rd, Melton LJ, 3rd, Chu CP. Diabetes mellitus and risk of skeletal fracture. N Engl J Med 1980; 303: 567-70.

[11] de L, II, van der Klift M, de Laet CE, et al. Bone mineral density and fracture risk in type-2 diabetes mellitus: the Rotterdam Study. Osteoporos Int 2005; 16: 1713-20.

[12] Strotmeyer ES, Cauley JA, Schwartz AV, et al. Nontraumatic fracture risk with diabetes mellitus and impaired fasting glucose in older white and black adults: the health, aging, and body composition study. Arch Intern Med 2005; 165: 1612-7.

[13] Barrett-Connor E, Holbrook TL. Sex differences in osteoporosis in older adults with non-insulin-dependent diabetes mellitus. JAMA 1992; 268: 3333-7.

[14] Schwartz AV, Sellmeyer DE, Strotmeyer ES, et al. Diabetes and bone loss at the hip in older black and white adults. J Bone Miner Res 2005; 20: 596-603.

[15] Bonds DE, Larson JC, Schwartz AV, et al. Risk of fracture in women with type 2 diabetes: the Women's Health Initiative Observational Study. J Clin Endocrinol Metab 2006; 91: 3404-10.

[16] Tontonoz P, Hu E, Graves RA, et al. mPPAR gamma 2: tissuespecific regulator of an adipocyte enhancer. Genes Dev 1994; 8: 1224-34.

[17] Beresford JN. Osteogenic stem cells and the stromal system of bone and marrow. Clin Orthop Relat Res 1989; 240: 270-80.

[18] Gimble JM, Robinson CE, Wu X, et al. Peroxisome proliferatoractivated receptor-gamma activation by thiazolidinediones induces adipogenesis in bone marrow stromal cells. Mol Pharmacol 1996; 50: $1087-94$.

[19] Okazaki R, Toriumi M, Fukumoto S, et al. Thiazolidinediones inhibit osteoclast-like cell formation and bone resorption in vitro. Endocrinology 1999; 140: 5060-5.

[20] Okazaki R, Miura M, Toriumi M, et al. Short-term treatment with troglitazone decreases bone turnover in patients with type 2 diabetes mellitus. Endocr J 1999; 46: 795-801. 
[21] Watanabe S, Takeuchi Y, Fukumoto S, et al. Decrease in serum leptin by troglitazone is associated with preventing bone loss in type 2 diabetic patients. J Bone Miner Metab 2003; 21: 166-71.

[22] Lecka-Czernik B, Moerman EJ, Grant DF, et al. Divergent effects of selective peroxisome proliferator-activated receptor-gamma 2 ligands on adipocyte versus osteoblast differentiation. Endocrinology 2002; 143: 2376-84.

[23] Lecka-Czernik B, Gubrij I, Moerman EJ, et al. Inhibition of Osf2/Cbfa1 expression and terminal osteoblast differentiation by PPARgamma2. J Cell Biochem 1999; 74: 357-71.

[24] Tornvig L, Mosekilde LI, Justesen J, et al. Troglitazone treatment increases bone marrow adipose tissue volume in mice. Calcif Tissue Int 2001; 69: 46-50.

[25] Soroceanu MA, Miao D, Bai XY, et al. Rosiglitazone impacts negatively on bone by promoting osteoblast/osteocyte apoptosis. J Endocrinol 2004; 183: 203-16.

[26] Akune T, Ohba S, Kamekura S, et al. PPARgamma insufficiency enhances osteogenesis through osteoblast formation from bone marrow progenitors. J Clin Invest 2004; 113: 846-55.

[27] Wan Y, Chong LW, Evans RM. PPAR- $\gamma$ regulates osteoclastogenesis in mice. Nat Med 2007; 13: 1496-503.

[28] Schwartz AV, Sellmeyer DE, Vittinghoff E, et al. Thiazolidinedione use and bone loss in older diabetic adults. J Clin Endocrinol Metab 2006; 91: 3349-54.

[29] Grey A, Bolland M, Gamble G, et al. The Peroxisome proliferatoractivated receptor- $\gamma$ agonist rosiglitazone decreases bone formation and bone mineral density in healthy postmenopausal women: a randomized, controlled trial. J Clin Endocrinol Metab 2007; 92: 1305-10.

[30] Meymeh RH, Wooltorton E. Diabetes drug pioglitazone (Actos): risk of fracture. Canad Med Assoc J 2007; 177: 723-4.

[31] Colhoun HM, Betteridge DJ, Durrington PN, et al. Primary prevention of cardiovascular disease with atorvastatin in type 2 diabetes in the Collaborative Atorvastatin Diabetes Study (CARDS): multicentre randomised placebo-controlled trial. Lancet 2004; 364: 685-96.

[32] Collins R, Armitage J, Parish S, et al. MRC/BHF Heart Protection Study of cholesterol-lowering with simvastatin in 5963 people with diabetes: a randomised placebo-controlled trial. Lancet 2003; 361: 2005-16.

[33] Song C, Guo Z, Ma Q, et al. Simvastatin induces osteoblastic differentiation and inhibits adipocytic differentiation in mouse bone marrow stromal cells. Biochem Biophys Res Commun 2003; 308: 458-62.

[34] Ohnaka K, Shimoda S, Nawata H, et al. Pitavastatin enhanced BMP-2 and osteocalcin expression by inhibition of Rho-associated kinase in human osteoblasts. Biochem Biophys Res Commun 2001; 287: 337-42.

[35] Sugiyama M, Kodama T, Konishi K, et al. Compactin and simvastatin, but not pravastatin, induce bone morphogenetic protein-2 in human osteosarcoma cells. Biochem Biophys Res Commun 2000; 271: 688-92

[36] Woo JT, Kasai S, Stern PH, et al. Compactin suppresses bone resorption by inhibiting the fusion of prefusion osteoclasts and disrupting the actin ring in osteoclasts. J Bone Miner Res 2000; 15: 650-62.

[37] Oxlund H, Dalstra M, Andreassen TT. Statin given perorally to adult rats increases cancellous bone mass and compressive strength. Calcif Tissue Int 2001; 69: 299-304.

[38] Oxlund H, Andreassen TT. Simvastatin treatment partially prevents ovariectomy-induced bone loss while increasing cortical bone formation. Bone 2004; 34: 609-18

[39] Pasco JA, Kotowicz MA, Henry MJ, et al. Statin use, bone mineral density, and fracture risk: Geelong Osteoporosis Study. Arch Intern Med 2002; 162: 537-40.

[40] Chan KA, Andrade SE, Boles M, et al. Inhibitors of hydroxymethylglutaryl-coenzyme A reductase and risk of fracture among older women. Lancet 2000; 355: 2185-8.

[41] Schoofs MW, Sturkenboom MC, van der Klift M, et al. HMG-CoA reductase inhibitors and the risk of vertebral fracture. J Bone Miner Res 2004; 19: 1525-30.

[42] Rejnmark L, Olsen ML, Johnsen SP, et al. Hip fracture risk in statin users a population-based Danish case-control study. Osteoporos Int 2004; 15: 452-8.
[43] Rejnmark L, Vestergaard P, Mosekilde L. Statin but not non-statin lipid-lowering drugs decrease fracture risk: a nation-wide casecontrol study. Calcif Tissue Int 2006; 79: 27-36.

[44] Scranton RE, Young M, Lawler E, et al. Statin use and fracture risk: study of a US veterans population. Arch Intern Med 2005; 165: 2007-12.

[45] De Leo V, Morgante G, la Marca A, et al. Combination of statins and hormone replacement therapy in postmenopausal women is associated with increased bone mineral density. Gynecol Endocrinol 2003; 17: 329-32.

[46] LaCroix AZ, Cauley JA, Pettinger M, et al. Statin use, clinical fracture, and bone density in postmenopausal women: results from the Women's Health Initiative Observational Study. Ann Intern Med 2003; 139: 97-104.

[47] Bauer DC, Mundy GR, Jamal SA, et al. Use of statins and fracture: results of 4 prospective studies and cumulative meta-analysis of observational studies and controlled trials. Arch Intern Med 2004; 164: 146-52.

[48] Rejnmark L, Buus NH, Vestergaard P, et al. Effects of simvastatin on bone turnover and BMD: a 1-year randomized controlled trial in postmenopausal osteopenic women. J Bone Miner Res 2004; 19: 737-44.

[49] Nakashima A, Nakashima R, Ito T, et al. HMG-CoA reductase inhibitors prevent bone loss in patients with Type 2 diabetes mellitus. Diabet Med 2004; 21: 1020-4.

[50] Chung YS, Lee MD, Lee SK, et al. HMG-CoA reductase inhibitors increase BMD in type 2 diabetes mellitus patients. J Clin Endocrinol Metab 2000; 85: 1137-42.

[51] Safaei H, Janghorbani M, Aminorroaya A, et al. Lovastatin effects on bone mineral density in postmenopausal women with type 2 diabetes mellitus. Acta Diabetol 2007; 44: 76-82.

[52] Braatvedt GD, Bagg W, Gamble G, et al. The effect of atorvastatin on markers of bone turnover in patients with type 2 diabetes. Bone 2004; 35: 766-70.

[53] Maeda T, Matsunuma A, Kurahashi I, et al. Induction of osteoblast differentiation indices by statins in MC3T3-E1 cells. J Cell Biochem 2004; 92: 458-71.

[54] Ghosh-Choudhury N, Mandal CC, Choudhury GG. Statin-induced Ras activation integrates the phosphatidylinositol 3-kinase signal to Akt and MAPK for bone morphogenetic protein-2 expression in osteoblast differentiation. J Biol Chem 2007; 282: 4983-93.

[55] Jinnouchi Y, Yamagishi S, Takeuchi M, et al. Atorvastatin decreases serum levels of advanced glycation end products (AGEs) in patients with type 2 diabetes. Clin Exp Med 2006; 6: 191-3.

[56] Yamagishi S, Nakamura K, Matsui T, et al. A novel pleiotropic effect of atorvastatin on advanced glycation end product (AGE)related disorders. Med Hypotheses 2007; 69: 338-40.

[57] Yang WS, Jeng CY, Wu TJ, et al. Synthetic peroxisome proliferator-activated receptor-gamma agonist, rosiglitazone, increases plasma levels of adiponectin in type 2 diabetic patients. Diabetes Care 2002; 25: 376-80.

[58] Libby P, Plutzky J. Inflammation in diabetes mellitus: role of peroxisome proliferator-activated receptor-alpha and peroxisome proliferator-activated receptor-gamma agonists. Am J Cardiol 2007; 99: 27B-40B.

[59] Nissen SE, Wolski K. Effect of rosiglitazone on the risk of myocardial infarction and death from cardiovascular causes. N Engl J Med 2007; 356: 2457-71.

[60] Lago RM, Singh PP, Nesto RW. Congestive heart failure and cardiovascular death in patients with prediabetes and type 2 diabetes given thiazolidinediones: a meta-analysis of randomized clinical trials. Lancet 2007; 370: 1129-36.

[61] Erdmann E, Dormandy JA, Charbonnel B, et al. The effect of pioglitazone on recurrent myocardial infarction in 2,445 patients with type 2 diabetes and previous myocardial infarction: results from the PROactive (PROactive 05) Study. J Am Coll Cardiol 2007; 49: 1772-80.

[62] Lincoff AM, Wolski K, Nicholls SJ, et al. Pioglitazone and risk of cardiovascular events in patients with type 2 diabetes mellitus: a meta-analysis of randomized trials. JAMA 2007; 298: 1180-8. 